\title{
COUNTING OF RBC AND WBC USING IMAGE PROCESSING: A
}

\section{REVIEW}

\author{
Akshaya P. Sahastrabuddhe ${ }^{1}$ \\ ${ }^{\text {I}}$ PG Research Student, Electronics and Tele-Communication Engineering, Maharashtra Institute of Technology, \\ Aurangabad, Maharashtra, India.
}

\begin{abstract}
The human blood consists of the RBCs, WBCs, Platelets and Plasma. The complete blood count defines the state of health. Blood is a health indicator therefore segmentation and identification of blood cells is very important. Complete Blood Count (CBC) includes counting of all the cells which determines person's health. The RBC and WBC count is very important to diagnose various diseases such as anemia, leukemia, tissue damage, etc. Old conventional method used in the hospital laboratories involves manual counting of blood cells using device called Hemocytometer and microscope. But this method extremely monotonous, laborious, time consuming, and leads to the inaccurate results due to human errors. Also there are some expensive machines like Analyzer, which are not affordable by every laboratories. The objective of this paper is to produce a survey on an image processing based system that can automatically detect and count the number of RBCs and WBCs in the blood sample image. Image Acquisition, Pre-Processing, Image Enhancement, Image Segmentation, Image Post-Processing and Counting algorithm these are six steps involved in an image processing algorithm. The objective of this research is to study the different methodologies of cells counting.
\end{abstract}

Ke words: RBC, WBC, Platelets, Digital Image Processing, Morphology, Hough Transform.

\section{INTRODUCTION}

The complete blood count $(\mathrm{CBC})$ is the blood test used to evaluate the health of person and to detect the disorders like anemia, infection and leukemia. In medical diagnosis Complete blood count is very important [1]. There are mainly four categories of cells:Red Blood Cells (RBCs), White Blood Cells (WBCs), Platelets and Plasma. These groups can be differentiated using texture, color, size, and morphology of nucleus and cytoplasm. Cells count is important to determine the immunity and capability of the body system. The abnormal count of cells indicates the presence of disease and person needs medical help[2].

Current research is on an implementation of image processing based automated counting of RBCs and WBCs from blood image.

WBCs are also called leukocytes. These cells are an important part of immune system. These protects body by removing viruses and bacteria in a body. Medical term use to describe low count is Leukopenia. Leukopenia indicate the presence of infection. Medical term use to describe high count is Leukocytosis. Leukocytosis indicate an existence of infection, leukemia or tissue damage.

$\mathrm{RBC}$ are also known as erythrocytes. The function of RBC is to carry oxygen and collects carbon dioxide from a lungs to the cells of body. They contains protein called hemoglobin. The presence of inner and outer layers of protein gives red color to blood. Hemoglobin do the work of carrying oxygen. An abnormal count of RBCs lead to anemia which results in mental tiredness, illness, weakness, dizziness. If it is not treated immediately it results into more serious symptoms like malnutrition and leukemia. RBC indices gives information about size and shape of cells and are also useful in differentiating types of anemia.

Platelets are also called as thrombocytes. The function of the platelets is to stop bleeding by clumping and clotting blood vessel injuries. A low platelet count is called thrombocytopenia. It avoids blood clotting and causes a person to bleed. High platelet count is called thrombocytosis. It clots blood inside blood vessel and stops blood from flowing properly [3]. Therefore for proper blood flow platelets count must be in normal range.

As mentioned earlier, $\mathrm{CBC}$ include counting of $\mathrm{RBCs}$, WBCs, Platelets, Hematocrit and Hemoglobin component in the blood cell. Table 1 shows the standard CBC for healthy person [1].

Table -1: Standard CBC for healthy person

\begin{tabular}{|l|l|l|l|}
\hline $\begin{array}{l}\text { Blood cell } \\
\text { type }\end{array}$ & Women & Men & Unit \\
\hline RBC & $4-5$ & $4.5-6.0$ & $\mathrm{M} / \mu \mathrm{L}$ \\
\hline WBC & $4.5-11$ & $4.5-11$ & $\mathrm{~K} / \mu \mathrm{L}$ \\
\hline Platelets & $150-450$ & $150-450$ & $\mathrm{~K} / \mu \mathrm{L}$ \\
\hline Hematocrit & $36 \%$ to $45 \%$ & $42 \%$ to $50 \%$ & $\%$ \\
\hline Hemoglobin & $12-15$ & $14-17$ & $\begin{array}{l}\mathrm{gm} / 100 \\
\mathrm{ml}\end{array}$ \\
\hline
\end{tabular}

(Units:- M-Million, K-Thousand, $\mu$ L-microliter, gm- grams, ml- milliliter, gm/100ml- gram per 100 milliliter.) 


\section{TRADITIONAL APPROACH}

The conventional method used to count blood cells involves counting by Hemocytometer. This device was specially designed for the complete blood count. Louis-Charles Malassez invented this hemocytometer. It consists of a chamber of certain dimensions. This chamber is created by a rectangular thick glass microscope slide. This chamber have grid of perpendicular lines etched on it. For counting purpose, person have to view Hemocytometer through a microscopeand count blood cells using hand tally counter [4]. The depth of the chamber and area bounded by perpendicular lines is known. Therefore it is possible to count the number of cells present in a specific volume of fluid, and thereby calculate the concentration of cells in the fluid overall [5].

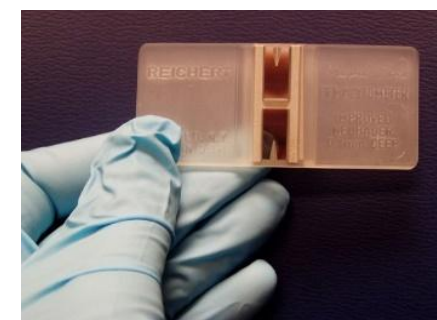

Fig -1: A Hemocytometer

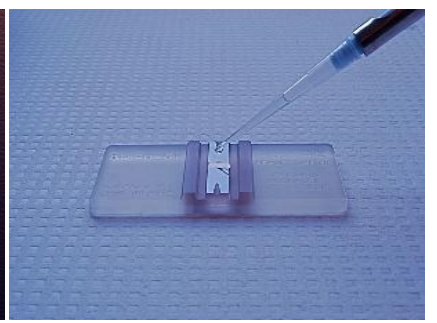

Fig -2: Load a Chamber

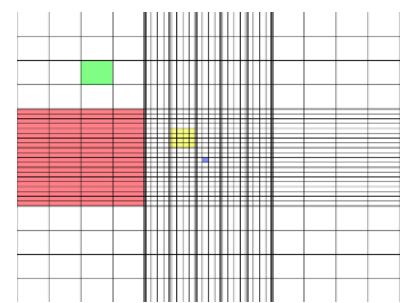

Fig -3: Hemocytometer Grid

Table 2: Hemocytometer grid details

\begin{tabular}{|c|c|c|}
\hline $\begin{array}{l}\text { Dimensions } \\
\text { (in } \mathrm{mm} \text { ) }\end{array}$ & $\begin{array}{l}\text { Area } \\
\left.\mathrm{mm}^{2}\right)\end{array}$ & $\begin{array}{l}\text { Volume at } 0.1 \mathrm{~mm} \\
\text { depth (in } \mathrm{nL} \text { ) }\end{array}$ \\
\hline $1 \mathrm{X} 1$ & 1 & 100 \\
\hline $0.25 \times 0.25$ & 0.0625 & 6.25 \\
\hline $0.25 \times 0.20$ & 0.05 & 5 \\
\hline $0.20 \times 0.20$ & 0.04 & 4 \\
\hline $0.05 \times 0.05$ & 0.025 & 0.25 \\
\hline
\end{tabular}

(Units: - mm- millimeter, nL- nano liter)

In the manual WBC count, 950 $\mu$ l dilution solution is mixed with $50 \mu \mathrm{l}$ of blood. The dilution ratio is of $1: 20$. The WBC nucleus is stained. Then the counting chamber is immediately filled with this mixture of dilution solution and blood. After few minutes, the counting of the WBCs begins. This counting is done in the 4 large squares.

Formula for WBC count:

WBC $($ in count $/ \mu \mathrm{l})=$

Number of WBCs counted $\times$ dilution factor

Number ofsquares $\times$ volume above a big square
In a manual count of RBC, $10 \mu \mathrm{l}$ of blood is mixed with $2000 \mu$ l of dilution solution. i.e dilution ratio is $1: 200$. Then counting chamber is immediately filled with well mixed dilution solution and blood. After 3-4 minutes, the RBCs will have settled, and the counting the RBCs begins. This counting is done in 80 small squares.

Formula for RBC count:

$\mathrm{RBC}($ in count $/ \mu \mathrm{l})=$

Number of RBCs counted $\times$ dilution factor

Number of squares $\times$ volume above a small square

Drawbacks of the manual method:

a). It is time-consuming and laborious.

b).Counting overlapping blood cells is a major problem.

c). It is difficult to get accurate results from visual inspection.

Many of the researchers have worked on the blood cell images and it's counting methods with the use of image processing. Those works are reviewed to get brief idea about the different methods in the area. The conclusion from this paper is hoped to be useful for beginners to build an algorithm to perform blood cell counting using image processing method.

\section{IMAGE PROCESSING APPROACH}

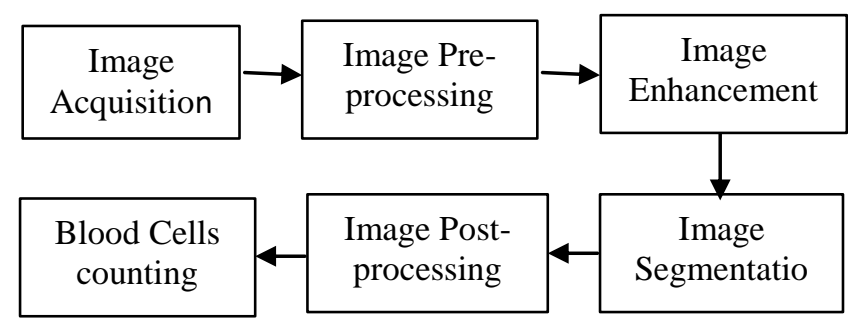

Fig- 4: General Block Diagram

These are six major steps involved in blood cells estimation.

\subsection{Image Acquisition}

Image acquisition acquires digital images of blood samples in either .jpeg or .png format. These images are in RGB color plane. These are microscopic image that are obtained from hospitals or from laboratories using digital microscopes or using a digital camera placed at the eye piece of a microscope. Images are also available on online medical library[6]. To examine the RBCs and WBCs stained blood images may be captured with the help of thin glass slides and Digital microscope [7]. Giesma stained thin blood film image should be taken so that platelets, RBCs and WBCs can be easily distinguished. In [8]to differentiate RBCs from WBCs and Platelets, RBCs are less stained as compare to WBCs and platelets leaving a bright patch with intensity value similar to background value. These images are digital images in either .jpeg or .png format and are in RGB color plane. 


\subsection{Image Pre-Processing}

It is a technique of adjusting images, improving the quality of image and making them suitable for the next step of process. Image pre-processing usually includes removing noise, contrast enhancing, isolating regions and use of different color models grayscale image [3] and HSV image [4], [11], Binarization. Grayscale represents the intensity of the image. As acquired images have low contrast and due to clustered white blood cells noise get included. To overcome and reduce these effects contrast enhancement is done [6]. After contrast stretching image is converted into grayscale, noise get added into resultant image is salt $\mathrm{n}$ paper noise. Also at the time of capturing the microscopic blood images noise get added into it, Median filtering is used to remove noise [3], [10]. After observing various sample images it was found that median filter would be the best noise removal filter [2].

\subsection{Image Enhancement}

Enhancement techniques improves the quality, contrast and brightness characteristics of an image, also sharpen its details. Histogram plotting, histogram equalization, image negation, image subtraction and filtering techniques, etc. are basic Image enhancement techniques.

In [11] Hue saturation is used for enhancing an image. The histogram thresholding is used to distinguish the nucleus of the leukocyte or WBCs from the rest of the cells in the image [12]. To get enhanced image, pre-processing is done to get enhanced image with Contrast-Limited Adaptive Histogram Equalization is used by Haider Adnan Khan et al. [13]. As the green color plane contains more information about the image as compare to blue and red color plane. Green color plane is extracted. To enhance the image, its contrast is adjusted by plotting its histogram [4]. In [4] canny edge detection and connected component labelling is used asimage enhancement techniques. The goal of edge detection is to extract the important features like line, corners, curves etc. from the edge of animas.

\subsection{Image Segmentation}

The segmentation is used to separate object from the background. Different segmentation methods are segmentation by using Histogram Thresholding, Otsu Adaptive Thresholding [3], Global Thresholding [4], Hough Transform [2] and Watershed transform algorithm [22], as well as by K- Means Clustering, nucleus segmentation by Gram-Schmidt Orthogonalization and a snake algorithm. The Circular Hough Transform detects some unnecessary circles due to overlapping, therefore removing one of the overlapping circles and then taking average of the count of both the method provides very accurate results [2], [15], [16]. Circular Hough transform is a frequently used method for detecting circles in an image, it often suffers from degradation in performance, especially in terms of speed, because of the large amount of edges given by complex background or texture [23], [24]. Naveed Abbas [25] et al.modified Hough Transform, it was proposed to improve the detection of low-contrast circular objects.
Some researchers use segmentation to classify abnormalities in the cells. In analysis of WBC, to segment the nucleus some techniques used are Gradient Vector Flow (GVF), snake algorithm and Zack Thresholding [17]. Also a Fuzzy approach is proposed for classified pixels to Region of Interest (ROI) [18]. Fuzzy C- Means (FCM) Clustering is used for sub image component [19]. The same work by using sub image component for feature space clustering is done [20]. To classify WBC automatically another advance work also is done in term of calculating area, major axis length over minor axis length, perimeter, circularity and ratio of areas between nucleus and cytoplasm [21].

\subsection{Image Post -Processing}

Image post processing includes Feature extraction and morphological operations.

Morphology includes dilation, erosion, granulometry and morphological filtering. Closing operation is used to fill the holes and gaps and opening operation is used to smoothen an image [9], [11]. Different types of structuring of elements are there for dilation and erosion. In [8] the concentric ring is used for dilation and a disk for erosion. Morphological operations eventually removes platelets and other stained parasites. In [26] author used morphological area closing to lower pixel value image and dilation and area closing to higher pixel value image.

Feature extraction extracts features that contain quantitative information about objects of interest. Shape features are: geometric parameters like areas, cell perimeter, and ratio of nucleus to overall cell area, boundary of the nucleus and circularity factor [17]. Texture features are entropy, homogeneity and contrast. Color features includes color histogram, mean and standard deviation of the color components in CIE-Lab domain [10]. Circular Hough Transform (CHT) is technique used in image analysis to detect the objects in circular form [15].Classifiers likeNearest Neighbor (NN), K-Nearest Neighbor (KNN), W-KNN, Bayes, Support Vector Machine (SVM), Neural Network (NNet) [27], Artificial Neural Networks (ANNs) [9],[28] and Local Linear Map (LLM), Fuzzy Cellular Neural Networks are included in feature extraction and are often used to classify blood cells.

Before doing labelling, borders are removed. Cells containing borders contain less information. Therefore, borders has to be removed to reduce complexity [10], [16] used 'imclear' function to clear the borders in an image.

\subsection{Blood Cells Counting}

Counting algorithm is applied to measure number of RBCs and WBCs. The most popular method used for counting is connected component labeling. Counting of RBCs and WBCs is done by finding number of connected components in segmented image [14]. It labels the connected objects in an image [3], [4], [11]. P. S. Hiremath et al. [17] used these labels for the subsequent feature extraction procedure. CHT is also a popular method for counting RBCs in an image. CHT counts number of circular objects i.e. RBCs in an image [4], [11] [15]. 
Table 3: Summary table

\begin{tabular}{|c|c|c|c|c|}
\hline \multicolumn{3}{|c|}{ Techniques } & Reference no. & Description \\
\hline \multicolumn{5}{|c|}{ Image Segmentation } \\
\hline 1 & \multicolumn{2}{|c|}{ Watershed algorithm } & {$[1],[11],[22]$} & Used to separate overlapping cells. \\
\hline 2 & \multicolumn{2}{|c|}{ Hough Transform } & {$[2],[4],[14],[15],[16],[23],[25],[26]$} & $\begin{array}{l}\text { Complex, require more processing time. } \\
\text { provides more accurate segmentation }\end{array}$ \\
\hline 3 & \multicolumn{2}{|c|}{ Otsu thresholding } & {$[4],[3]$} & $\begin{array}{l}\text { Automatically perform clustering - based } \\
\text { image thresholding }\end{array}$ \\
\hline 4 & \multicolumn{2}{|c|}{ Grey Thresholding } & {$[3],[10],[11],[13],[14]$} & \\
\hline 5 & \multicolumn{2}{|c|}{ K-mean clustering } & {$[23],[25]$} & It is fast method \\
\hline 6 & \multicolumn{2}{|c|}{ Fuzzy C-means clustering } & [18], [19] & \\
\hline 7 & \multicolumn{2}{|c|}{ Holes filling } & {$[3],[11]$} & Results in smooth image \\
\hline 8 & \multicolumn{2}{|c|}{ Angular Ring Ratio (ARR) } & {$[8]$} & \\
\hline \multicolumn{5}{|c|}{ Image Post-processing } \\
\hline 1 & \multicolumn{2}{|c|}{ Morphology } & {$[8],[9],[10],[14],[16]$} & Opening and closing \\
\hline 2 & \multicolumn{2}{|c|}{ Feature Extraction } & {$[7],[17],[27],[28]$} & Extracts quantitative information \\
\hline & $\mathrm{i}$ & Shape features & [9], [10] & $\begin{array}{l}\text { Features are: Area, perimeter, boundary and } \\
\text { circularity factor }\end{array}$ \\
\hline & ii & Texture features & {$[10]$} & $\begin{array}{l}\text { Features are: Entropy, Contrast, and } \\
\text { Homogeneity. }\end{array}$ \\
\hline & iii & Color Features & {$[10]$} & $\begin{array}{l}\text { Features are: Color histogram, Mean and } \\
\text { Standard deviation }\end{array}$ \\
\hline & iv & Classifiers & [17], [27], [28] & $\begin{array}{l}\text { NN, KNN, W-KNN, Bayes, SVM, NNet, } \\
\text { ANN }\end{array}$ \\
\hline 3 & Bor & emoving & [3], [10], [11], [16] & $\begin{array}{l}\text { Borders contains no information. To reduce } \\
\text { the complexity borders has to be removed }\end{array}$ \\
\hline \multicolumn{5}{|c|}{ Cells counting } \\
\hline 1 & \multicolumn{2}{|c|}{ Labelling connecting components } & [3], [4], [10], [11], [17] & It labels the connected objects. \\
\hline 2 & \multicolumn{2}{|c|}{ Circular Hough transform } & {$[4],[11],[15],[16]$} & Counts number of circles in image \\
\hline
\end{tabular}

\section{CONCLUSION}

This paper presents a review on software based solutions for counting the blood cells. Image processing based method of cell counting is fast, cost effective and produces accurate results. MATLAB software is used for the analysis. The accuracy of a system depends upon the quality of input image, Camera used for acquiring an image.

\section{ACKNOWLEDGEMENT}

I am grateful to my guide Dr. Sayyad D. Ajij for providing me with invaluable guidance in this research.

\section{REFERENCES}

[1]. J. M. Sharif, M. F. Miswan, M. A. Ngadi andMdSahHj "Red Blood Cell Counting Using Masking And Watershed Algorithm: A Preliminary Study", 2012 International Conference on Biomedical Engineering, Penang, Malaysia, February 2012.

[2]. Pawan Agrawal andPradiptiVarma "Automated Detection and Counting of Red Blood Cell using Image Processing Techniques", International Journal of scientificresearch and management, Vol. 3, Issue 4, pp. 2692-2695, 2015.

[3]. Pooja R. Patil, G. S. Sable and GauriAnandgaonkar,
"Counting of WBCs and RBCs from Blood Images Using Gray Thresholding”, International Journal of Research in Engineering and Technology, Vol. 03, Issue: 04, April2014.

[4]. Thejashwini M and M C Padma "Counting of RBCs And WBCs using Image Processing Techniques",International Journal on Recent and Innovation Trends in Computing and Communication, Vol. 3 Issue: 5, May 2015.

[5]. Oscar Bastidas, "Cell Counting with Neubauer Chamber Basic Hemocytometer Usage", Technical Note - Neubauer Chamber Cell Counting.

[6]. AmrutaPandit, ShrikrishnaKolhar andPragatiPatil "Survey on Automatic RBC Detection and Counting", International Journal of Advanced Research in Electrical, Electronics and Instrumentation Engineering, Vol. 4, Issue: 1,January 2015.

[7]. Naveen Jambhekar, "Red Blood Cells Classification using Image Processing", Science Research Report 1(3): pp. 151-154, November 2011

[8]. S. Kareem, R.C.S Morling and I. Kale, "A Novel Method to Count the Red Blood Cells in Thin Blood Films", IEEE International Symposium on circuits and systems, pp. 1021 - 1024, May - June 2011.

[9]. Madhuri G. Bhamare and D. S. Patil, "Automatice Blood Cell AnalysisbyUsing Digital ImageProcessing: 
A PreliminaryStudy", International Journal of EngineeringResearch\&Technology, Vol. 2,Issue: 9, September 2013.

[10].MojtabaTaherisadr, MonaNasirzonouzi, BehzadBaradaran andAlirezaMehdizade, "New Approach to Red Blood Cell Classification Using Morphological Image Processing", Shiraz E-Medical Journal, Vol. 14, January 2013.

[11].SumeetChaurasiya and G Usha Rani, "Automatic Red Blood Cell Counting using Watershed Segmentation", International Journal of ComputerScience and Information Technologies, Vol. 5, pp. 4834-4838, 2014.

[12].Roy A. Dimayuga, Gerwin T. Ong, Rainier Carlo S. Perez, Gefferson O. Siy, Saman C. SohrabiLangroudi and Miguel O.Gutierrez. "Leukemia DetectionUsing Digital ImageProcessing in Matlab", ECE StudentForum, De La Salle University, Manila. March 2010.

[13].Haider Adnan Khan, GolamMorshedMaruf, "Counting Clustered Cells using Distance Mapping," International Conference on Informatics, Electronics and Vision, pp. 1-6, May 2013.

[14].Vinutha H Reddy, "Automatic Red Blood Cell and White Blood Cell Counting For Telemedicine System", International Journal of Research in Advent Technology, Vol. 2, Issue: 1, January 2014.

[15]. MausumiMaitra, Rahul Kumar Gupta and Manali Mukherjee, "Detection and Counting of Red Blood Cells in Blood Cell Images using Hough Transform", International Journal of Computer Applications, Vol. 53, September 2012.

[16]. SitiMadihahMazalan, NasrulHumaimiMahmood and MohdAzhar Abdul Razak, "Automated Red Blood Cells Counting in Peripheral Blood Smear Image Using Circular Hough Transform", First IEEE International Conference on Artificial Intelligence, Modelling \& Simulation, pp. 320 - 324, 2013.

[17].P. S. Hiremath , ParashuramBannigidadandSaiGeeta, "Automated Identification and Classification of White Blood Cells (Leukocytes) in Digital Microscopic Images", ", International Journal of Computer Applications, Special Issue on -Recent Trends in Image Processing and Pattern Recognition\| , 2010.

[18].Nasution, AMT and EK Suryaningtyas, "Comparison of Red Blood Cells Counting using two Algorithms: Connected Component Labeling and Back projection of Artificial Neural Network", IPGC 2008, IEEE, 2008.

[19].K.S. Kim, P.K. Kim and J.J. Song, "Analysing Blood Cell Image to Distinguish Its Abnormalities", 8Th ACM international conference on Multimedia, 2000.

[20].Adnan Khashman andEsam Al-zgoul, "Image segmentation of blood cell in Leukemia patients", Recent Advances in Computer Engineering and Applications, N. CYPRUS, 2009.

[21].S. S. Savkare and S. P. Narote, "Automatic Classification of Normal and Infected Blood Cells for Parasitemia Detection", International Journal of Computer Science and Network Security, Vol. 11, February 2011.
[22].HemantTulsani, SaranshSaxena and Naveen Yadav, "Segmentation using Morphological Watershed Transformation for Counting Blood Cells", International Journal of Computer Applications \& Information Technology, Vol. 2, Issue: 3, Apr-May 2013.

[23].Monika Mogra, ArunBansel, Vivek Srivastava, "Comparative Analysis of Extraction and Detection of RBCs and WBCs Using Hough Transform and kMeans Clustering Algorithm", International Journal of Engineering Research and General Science Vol. 2, Issue: 5, pp. 670 - 674, August-September 2014

[24].Minal D. Joshi, Atul H. Karode, and S. R. Suralkar,"White Blood Cells Segmentation and Classification to Detect Acute Leukemia" , International Journal of Emerging Trends \& Technology in Computer Science, Vol. 2, May - June 2013.

[25].Naveed Abbas and DzulkifliMohamad, "Microscopic RGB color images enhancement for blood cells segmentation in ycbcr color space for $\mathrm{k}$-means clustering", Journal of Theoretical and Applied Information Technology, Vol. 55, september 2013.

[26].Venkatalakshmi. B and Thilagavathi. K, "Automatic Red Blood Cell Counting Using Hough Transform”, Proceedings of IEEE Conference on Information and Communication Technologies, pp.268-270, 2013.

[27].Fabio Scotti, “Automatic Morphological Analysis for Acute Leukemia Identification in Peripheral Blood Microscope Images,ICoCIMSA 2005 - IEEE, 2005

[28].Seyed Hamid Rezatofigh, KosarKhaksari, and Hamid SoltanianZadeh, "Automatic recognition of five types of white blood cells in peripheral blood", 7th International Conference, ICIAR 2010, Póvoa de Varzim, Portugal, June 2010, 